\title{
Local Domain Wall Structure in Polycrystalline $\mathrm{BiFeO}_{3}$
}

A. Bencan $^{1}$, G. Drazic ${ }^{2}$, H. Ursic ${ }^{1}$, N. Sakamoto ${ }^{3}$, B. Jancar ${ }^{4}$, N. Wakiya ${ }^{5}$, H. Suzuki ${ }^{3}$, B. Malic ${ }^{1}$, D. Damjanovic $^{6}$ and T. Rojac ${ }^{1}$

1. Electronic Ceramics Department, Jozef Stefan Institute, Ljubljana, Slovenia.

2. Laboratory for Materials Chemistry, National Institute of Chemistry, Ljubljana, Slovenia.

3. Research Institute of Electronics, Shizuoka University, Hamamatsu, Japan.

4. Advanced Materials Department, Jozef Stefan Institute, Ljubljana, Slovenia.

5. Graduate school of Science and Technology, Shizuoka University, Hamamatsu, Japan.

6. Ceramics Laboratory, Swiss Federal Institute of Technology - EPFL, Switzerland.

In multiferroic $\mathrm{BiFeO}_{3}(\mathrm{BFO})$, the domain walls have properties that are fundamentally different from those of the bulk, e.g., it was shown that the domain walls in BFO thin films possess higher electrical conductivity than the surrounding [1]. While detailed structural studies of domain walls were performed on BFO thin films, these issues are only at their early research stage for the bulk BFO ceramics. This is because the preparation of $\mathrm{BFO}$ ceramics is non-trivial; $\mathrm{Bi}_{2} \mathrm{O}_{3}$ volatility, variation in Fe valence state, possible reactions of BFO with impurity elements, lead to formation of undesired secondary phases [2].

Recently, we demonstrated that the local conductivity at domain walls influences the macroscopic properties of bulk BFO [3]. In order to make further steps in the understanding of the domain wall conductivity and electromechanical response of BFO ceramics, we investigate the local structure of domain walls using different microscopy methods. As opposed to epitaxial thin films, the determination of the type of the domain walls in polycrystalline ceramics with randomly oriented grains is practically impossible by using just AFM/PFM. By identical location approach, we were able to determine the type of individual domain walls in BFO ceramics on a micro level with the combination of SEM/EBSD and AFM/PFM analysis.

$\mathrm{BiFeO}_{3}$ ceramics were prepared by reactive sintering of a mechanochemically activated $\mathrm{Bi}_{2} \mathrm{O}_{3}-\mathrm{Fe}_{2} \mathrm{O}_{3}$ powder mixture at $760^{\circ} \mathrm{C}$ or $820^{\circ} \mathrm{C}$ in a packing powder. The ceramics had a relative density of $93 \%$.

The structure of domain walls in the BFO ceramics was analyzed by different scanning electron microscopy methods on a micro level (SEM, EBSD), down to atomic scale using atomically resolved Cs-probe corrected JEM-ARM200CF microscope.

In rhombohedral BFO ceramics, three different domain walls, i.e., $180^{\circ}$ on $\{111\}$ planes, $71^{\circ}$ on $\{110\}$ planes and $109^{\circ}$ on $\{100\}$ planes were observed. Experimentally, a combination of SEM/EBSD and cAFM studies shows that all types of domain walls, i.e., $71^{\circ}, 109^{\circ}$ and $180^{\circ}$, are conductive in BFO ceramics [Figure 1]. We precisely analyzed the local structure of domain walls at the atomic level by measuring the displacement of Fe cations within the periodic structure [Figure 2]. In this way, we were able to determine the local polarization direction, local variations in lattice parameters and potential local strains on the level of unit cells.

The complexity of domain wall structure will be discussed and related to the macroscopic, functional response of the polycrystalline BFO. 
References:

[1] J Seidel et al, Nat. Mater. 8 (2009), p.229

[2] T. Rojac et al, J. Am. Ceram. Soc. 94 (2014), p.1993

[3] T. Rojac et al, Adv. Funct. Mater. 2015, doi:10.1002/adfm.201402963

[4] This work was supported by the Slovenian Research Agency (P2-0105 and J2-5483).

a)

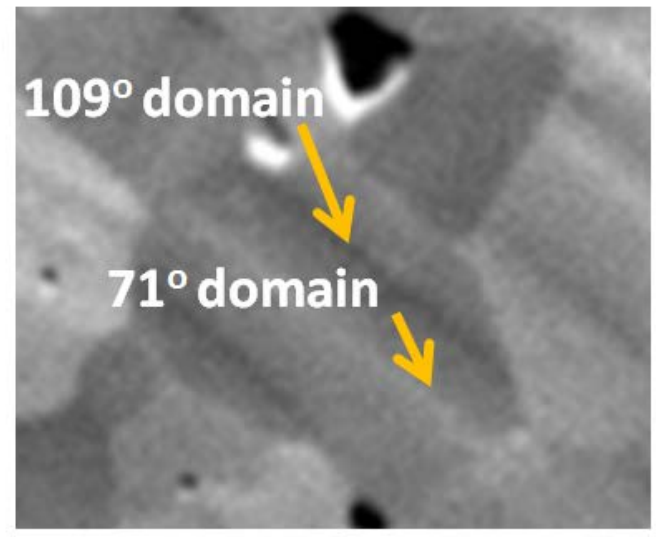

b)

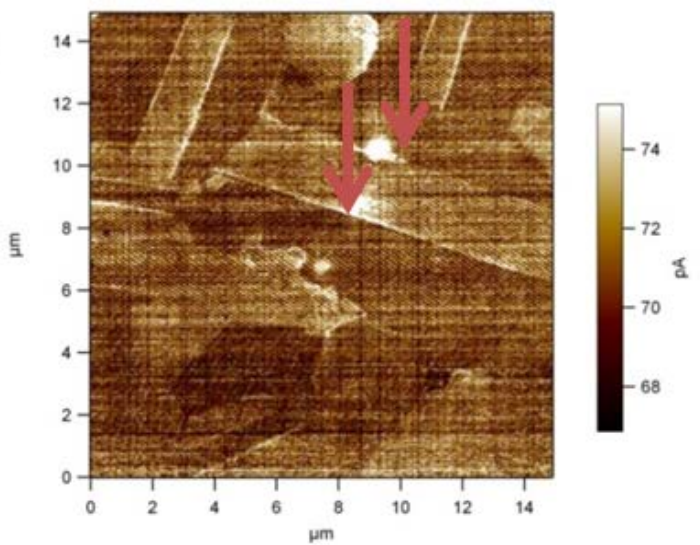

Figure 1. SEM (a) and corresponding electric current image (b) of $109^{\circ}, 71^{\circ}$ domain walls in $\mathrm{BiFeO}_{3}$ ceramics. The types of domain walls were identified by EBSD analysis. Electric current images at $+11 \mathrm{~V}$ showed higher electric current signal at domain walls as compared to that of the surrounding domains.

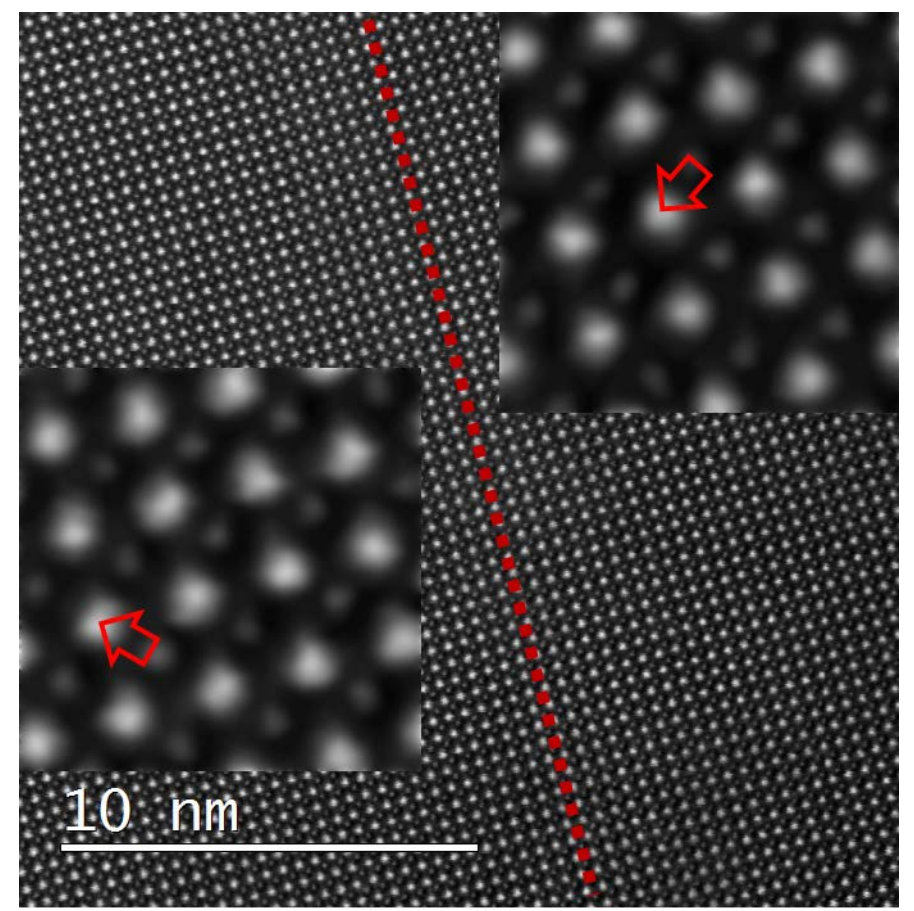

Figure 2. HAADF-STEM image of $109^{\circ}$ domain wall. Inserts show Fe displacement from the central position. 